\title{
The Psychological Benefits of Vigorous Exercise: A Study of Discordant MZ Twin Pairs
}

\author{
Wendy Johnson and Robert F. Krueger \\ Department of Psychology, University of Minnesota - Twin Cities, Minneapolis, United States of America
}

\begin{abstract}
T he physiological benefits of vigorous exercise are well established. The existence of psychological benefits is less clear, however, due to methodological limitations common to investigatory studies. Two of these limitations are the difficulty of establishing appropriate control groups and the large variety of highly specific measures of psychological function available for consideration of effects. To address these limitations, we identified 63 pairs of monozygotic twins from the National Survey of Midlife Development in the United States who were discordant for the amount of vigorous exercise in which they engaged regularly. The twins who regularly engaged in vigorous exercise experienced greater positive psychological functioning than their nonexercising co-twins as measured by the latent factor representing the variance common to 8 measures of mood, optimism, control over life, and interpersonal aspects of personality. The magnitude of the difference was in excess of 4 standard deviations.
\end{abstract}

The physiological benefits of exercise for purposes of both prevention and rehabilitation of chronic disease are well established (Fletcher et al., 1992; Haskell, 1984). At the popular level, exercise is strongly associated with positive psychological functioning as well. In spite of this, most people (perhaps 70\%) in developed, western populations do not engage in regular vigorous exercise (Brawley \& Rodgers, 1994; Hamdorf et al., 2002), and attrition rates in exercise programs are high (Raglin, 2001). For example, the authors of one study of an effective behavioral intervention in exercise program adherence were pleased to report reduction of 6-month drop-out rates from $63 \%$ to $39 \%$ (Annesi \& Unruh, 2004). This suggests that adherence may be driven by psychological benefits that are not experienced to the same degree by all participants. For example, there is evidence that angiotensin-converting enzyme (ACE) genotype may be associated with endurance performance and adherence to exercise programs even in the absence of differences in baseline levels and changes in physiological measures in response to exercise (Thompson et al., 2006). Understanding why and the extent to which there may be individual differences of this kind that contribute to exercise adherence is important in aging populations, given the substantial evidence for the importance of regular exercise in maintaining physical health. Moreover, there is growing evidence that links among cognitive decline, depression symptomatology, and deteriorating physical health in the elderly may be reversed or slowed by physical exercise (Colcombe et al., 2006; Dishman et al., 2006).

Indeed, it is challenging to summarize research findings in this area succinctly. There are many reasons for this, but they can be grouped along two lines. First, psychological benefits can be measured in various ways, and results tend to differ depending on the specific measure of positive psychological functioning used, the nature of the exercise engaged in, and the degree to which this level of exercise is habitual to the study participants. For example, findings of increases in positive mood are clearest when regular exercisers undertake vigorous exercise at a level with which they are familiar (Salmon, 2001). In contrast, strenuous exercise in sedentary people has generally been associated with increased negative mood or decreased positive mood (Petruzello et al., 1997). Perhaps because of this, findings of increases in positive mood tend to be clearer and stronger than findings of decreases in negative mood (McIntyre et al., 1990; Steptoe et al., 1993). In addition, clinical samples may not yield the same results as communitybased samples. For example, assessment of effects of exercise on psychopathological symptomatology in community samples is subject to floor effects when the sample has too little symptomatology to show improvement with exercise (Salmon, 2001). And studies of stress resistance show varying results depending on the nature and timing of the induced

Received 11 August, 2006; accepted 11 September, 2006.

Address for correspondence: Wendy Johnson or Robert F. Krueger, Department of Psychology, University of Minnesota, 75 East River Road, Minneapolis, MN 55455,USA. E-mail: john4350@umn.edu, krueg038@umn.edu 
stressor and the specific measure of response to stress used (Salmon, 2001). For example, there is evidence that, during exercise, cardiovascular responses to mental stress increase (Rouselle et al., 1995), though emotional responses to mental stress decrease (Girodo \& Pellegrini, 1976).

The variation in results and its diverse sources suggest that an approach to the measurement of positive psychological functioning that can summarize positive psychological functioning may help to clarify the nature and extent of its association with exercise. We employed such a measure in this study, making use of a latent variable tapping the variance common to eight measures of positive psychological functioning. Latent variables have substantial advantages in measurement of psychological constructs. They represent the systematic confluence of measured variables; as such, they represent unidimensional constructs in their purest form (Bollen, 1989). The observed variables serving as indicators of a latent variable all contain random and/or systematic measurement error, but the latent variable is free of this.

The second group of reasons for complexity in results of studies investigating the psychological effects of exercise involves the possibility of self-selection. Broadly stated, the effect of exercise on psychological functioning may not be causal. Rather, to the extent that exercise appears to confer psychological benefits, it is possible that some unmeasured constitutional or environmental variable distinguishes the people who exercise from those who do not in that the exercisers respond to the world in general - and to exercise in particular - more positively than the nonexercisers. More specifically, it is possible that people who choose to exercise vigorously and regularly tend to enjoy it, and everything else, more than those who do not choose to exercise. Perhaps they also experience more positive and less negative mood, less depression and anxiety, greater control over their lives, higher self-concept, and/or greater resistance to stress than those who resist exercising (Salmon, 2001).

It is of course not possible to measure exhaustively every variable that could exert such a causative influence. Studies of monozygotic (MZ) twins reared together in childhood but discordant for level of exercise in adulthood, however, can usefully minimize, though not completely eliminate, such possible effects. This is because such $\mathrm{MZ}$ twins are matched on genetic and childhood environmental background, and thus the nonexercising twins serve as controls for these kinds of variables in relation to their exercising cotwins. There is evidence that this is relevant: there appear to be both general familial and genetic influences on both the tendency to exercise (e.g., Perusse et al., 2003; Simonen et al., 2002) and the physiological response to exercise (Kartodihardjo \& Klissouras, 1976). MZ twins are also the same gender, which means that whatever effects of sex may be present are also controlled. The use of this design makes it more likely that any differences between exercising and nonexercising $\mathrm{MZ}$ co-twins in psychological measures can be attributed to the amount of exercise they get. We made use of such a sample of discordant MZ twin pairs in this study. Thus, the purpose of this study was to investigate the existence and magnitude of psychological benefits of habitual vigorous exercise, compensating for the limitations of previous studies by using a latent summary variable of positive psychological functioning in a sample of $M Z$ twin pairs discordant for level of exercise.

\section{Participants and Measures}

The MZ twin pairs discordant for level of exercise in this study came from the MacArthur Foundation Survey of Midlife Development in the United States (MIDUS). The base sample consists of 998 twin pairs distributed roughly according to population throughout the continental United States and ranging in age from 25 to $74(M=44.9, S D=12.1)$. In total, the sample includes $172 \mathrm{MZ}$ male pairs, $195 \mathrm{MZ}$ female pairs, 138 dizygotic (DZ) male pairs, $214 \mathrm{DZ}$ female pairs, 262 opposite-sex pairs, and 17 pairs with missing or indeterminate zygosity information. The sample is $58 \%$ female; about $92 \%$ listed their race as Caucasian. Additional description of the sample and its recruitment is given in Johnson and Krueger (2004) and Kessler et al. (2004).

The MIDUS participants completed a series of selfreport questionnaires including questions on health, lifestyle, and psychological functioning. Level of exercise was assessed in two questions (one for each season), 'During the summer/winter, how often do you engage in vigorous physical activity (e.g., running or lifting heavy objects) long enough to work up a sweat?'. Response choices were 'several times a week or more', 'about once a week', 'several times a month', 'about once a month', 'less than once a month', and 'never'. The responses to each question were coded so that they represented estimated numbers of exercise sessions per month: 'several times a week or more' was coded as 13.5 , 'about once a week' was coded as 4.5 , 'several times a month' was coded as 3, and so forth. The estimates for the two questions were averaged. The resulting variable had a mean of 6.2 , a median of 4.5 , a mode of 13.5 , and a standard deviation of 5.1. Overall, the sample members may have exercised slightly more than average, as only $55 \%$ said they exercised once a week or less. Still, only $26 \%$ said they exercised three times a week or more and $25 \%$ said they exercised about once a month or less. Because the variable was significantly correlated with age $(-.18, p<.001)$ and because $M Z$ twins share a common age and gender which can inflate their apparent similarity, we regressed the effects of age and gender from the exercise variable using the data from the full sample. There were no significant main effects of gender. 


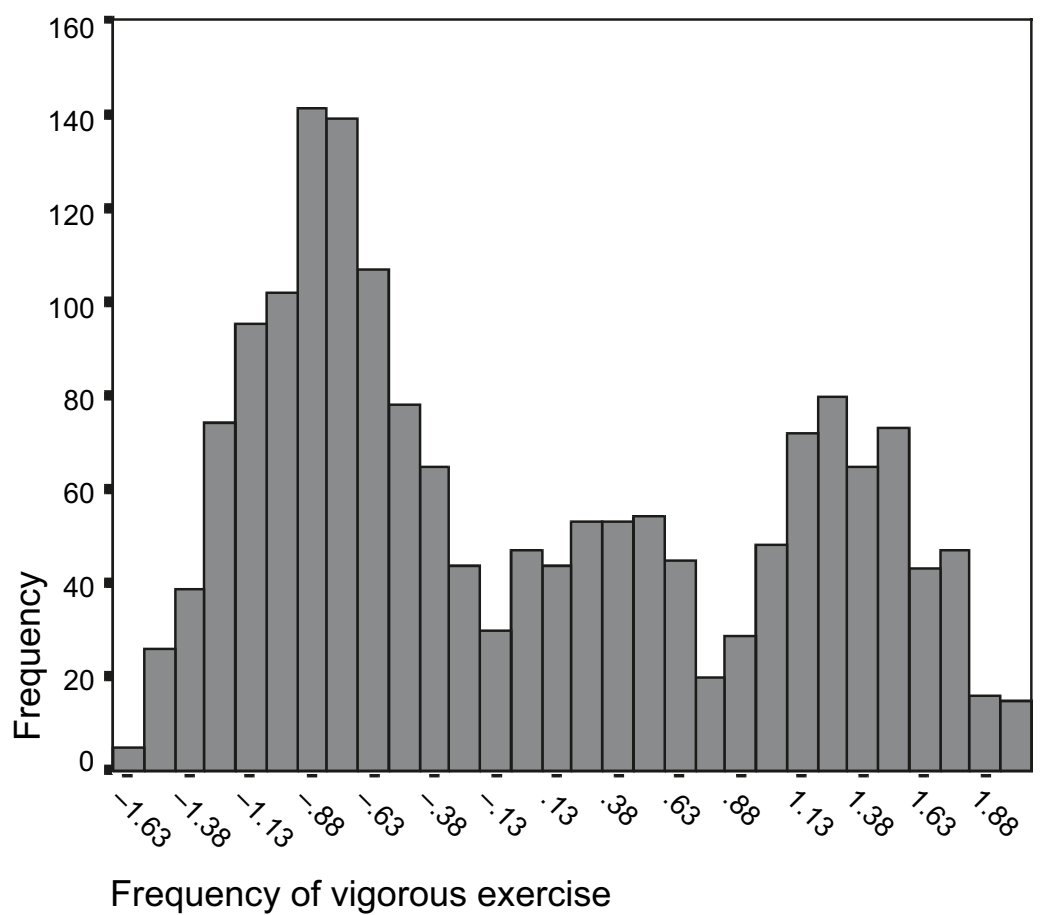

Figure 1

Distribution of frequency of vigorous exercise in full sample. The exercise variable was standardized following adjustment for the effects of age and gender.

The distribution of the resulting variable is shown in Figure 1. As the figure makes clear, the distribution was bi- or perhaps tri-modal. Most participants fell in the lower or upper ranges of the distribution. We made use of this in order to select MZ pairs functionally discordant for level of exercise by requiring that one member of each pair score below -.42 (in the bottom $47 \%$ of the distribution), while the other scored above .87 (in the top $28.5 \%$ of the distribution). This meant, roughly, that those we classed as nonexercisers exercised at most three times per month, while those we classed as exercisers exercised at least three times per week. There were 63 (34 male, 29 female) MZ twin pairs who met this discordance criterion (age range $25-73, M=44.7, S D=12.1$ ).

To measure psychological function, we made use of eight variables, adjusted to remove the effects of age and gender. We purposely selected trait measures of positive personality functioning that have shown substantial genetic influence (Bouchard \& Loehlin, 2001), as well as measures of positive attitude and mood that are more state-like in nature, because we were interested in constructing a latent variable that could transcend the questions raised by the existing literature regarding timing and continuity of effects. In addition, we wanted to include variables that show substantial genetic influence. Variables with substantial genetic influences should show substantial similarity in MZ twins, thus creating a base of similarity between $\mathrm{MZ}$ co-twins that should sharpen the effects of nonshared environmental influences relevant to the pairs' discordance for exercise, thus making stronger our test for such effects.

Participants quantified their experience of good mood by rating how much of the time in the past month they felt six forms of positive emotion (e.g., 'cheerful' and 'full of life') on a 5-point scale ranging from 'all the time' to 'none of the time'. Full-sample alpha for this scale was .91. Participants also indicated the extent to which the adjective 'optimistic' described them on a scale of 1 to 4 and rated their expected satisfaction with their lives 10 years in the future on a scale of 1 to 10 . We combined standardized scores on these two items to form a scale we called Optimism (alpha $=.41)$. The MIDUS questionnaires also included questions about the degree to which participants perceived they had control over various aspects of their lives, assessed on a scale from 1 to 10 . The questions read, 'How would you rate the amount of control you have over your __ these days?' and life aspects queried were health, work situation, financial situation, contribution to the welfare and well-being of other people, overall relationship with children, marriage or close relationship, sexual aspect, and life overall. Full-sample alpha for the scale formed from the mean response to these ratings was .73. Participants who did not have spouses, close relationships, or children and thus did not respond to these items received scores based on the mean of the items to which they did respond.

The remaining five variables we used assessed personality based on the Big Five Model (Extraversion, 
Agreeableness, Conscientiousness, Openness, and Neuroticism; Lachman \& Weaver, 1997), with the addition of a scale for Agency, a construct reflecting self-confidence and assertiveness. Each scale consisted of 4 to 7 adjectives chosen from existing trait lists and inventories (e.g., Goldberg, 1992; John, 1990; Trapnell \& Wiggins, 1990). Alphas for the scales in the pilot study used to develop them were $.74, .78$, $.80, .58, .77$, and .79 for Neuroticism, Extraversion, Agreeableness, Conscientiousness, Openness, and Agency respectively). For this study, because we were focusing on positive psychological functioning, we did not include the Neuroticism variable. There were no mean or variance differences between $M Z$ twin pairs discordant for exercise and the rest of the MIDUS twin sample on any of the eight measures of psychological function.

\section{Analysis and Results}

Table 1 shows the means and standard deviations for the eight measures of psychological functioning in exercisers and nonexercisers. As expected, with only one exception, exercisers showed more positive psychological functioning. Consistent with many results of studies of psychological benefits of exercise, however, the benefits did not appear to be consistent or clear-cut. Paired-sample $t$ tests showed at least marginally significant differences in five variables, but only two were significant $(p<.05)$ after Bonferroni correction for multiple testing. Stated so that positive statistics favor exercisers, effect sizes of differences ranged from -.05 (ns) for Optimism to $.44(p=.002)$ for Openness. The eight variables shared substantial common variance however, as suggested by the correlations among them shown in Table 2. In the full sample, factor analysis indicated that the first factor, with an eigenvalue of 3.33, explained $42 \%$ of the variance. The lowest loading on this factor was .44 from Conscientiousness. (By way of comparison, the second factor, with an eigenvalue of 1.09 , explained an additional $14 \%$ of the variance, and there were no additional factors with eigenvalues greater than 1.00 . In a two-factor solution, the factors were correlated .61.)

We used this single dominant common factor to construct a two-group (exercising $\mathrm{MZ}$ twins and nonexercising $\mathrm{MZ}$ twins) latent factor model with a

Table 1

Means, Standard Deviations, and Effect Sizes and Significance Levels of Differences in Psychological Variables Associated with Exercise in Exercising and Nonexercising MZ Twins

\begin{tabular}{|c|c|c|c|c|c|c|}
\hline \multirow[b]{2}{*}{ Variable } & \multicolumn{2}{|c|}{ Exercising $\mathrm{MZ}$ twins } & \multicolumn{2}{|c|}{ Nonexercising $\mathrm{MZ}$ twins } & \multirow{2}{*}{$\begin{array}{l}\text { Effect size of } \\
\text { pair difference }\end{array}$} & \multirow[t]{2}{*}{$p$ level } \\
\hline & Mean & $S D$ & Mean & $S D$ & & \\
\hline Good mood & 0.03 & 1.09 & -0.12 & 0.95 & 0.15 & .36 \\
\hline Optimism & -0.06 & 0.97 & -0.01 & 0.94 & -0.05 & .72 \\
\hline Control & 0.06 & 0.98 & -0.27 & 0.92 & 0.33 & .02 \\
\hline Agency & 0.12 & 1.00 & -0.15 & 0.92 & 0.27 & .06 \\
\hline Extraversion & 0.17 & 0.99 & -0.25 & 1.06 & 0.42 & .002 \\
\hline Agreeableness & 0.03 & 0.96 & -0.28 & 1.03 & 0.31 & .06 \\
\hline Conscientiousness & 0.05 & 1.09 & -0.06 & 0.91 & 0.11 & .36 \\
\hline Openness & 0.21 & 1.12 & -0.23 & 1.01 & 0.44 & .002 \\
\hline
\end{tabular}

Note: $M Z=$ monozygotic. $S D=$ standard deviation. There were 63 pairs of $M Z$ twins discordant forlevel of exercise. All variables are standardized in the full sample. $p$ levels are based on paired-samples $t$ tests, with no adjustment for the multiple tests performed. Effect sizes make use of the full-sample standard deviations, which were effectively 1.00 for all variables. They are positive when exercisers have higher mean levels.

\section{Table 2}

Correlations among Study Variables Associated with Exercise in Exercising and Nonexercising MZ Twins

\begin{tabular}{|c|c|c|c|c|c|c|c|c|}
\hline Variable & 1 & 2 & 3 & 4 & 5 & 6 & 7 & 8 \\
\hline 1. Good mood & 1.00 & & & & & & & \\
\hline 2. Optimism & .36 & 1.00 & & & & & & \\
\hline 3. Control & .49 & .41 & 1.00 & & & & & \\
\hline 4. Agency & .30 & .32 & .28 & 1.00 & & & & \\
\hline 5. Extraversion & .50 & .34 & .35 & .59 & 1.00 & & & \\
\hline 6. Agreeableness & .33 & .23 & .29 & .17 & .58 & 1.00 & & \\
\hline 7. Conscientiousness & .22 & .14 & .26 & .31 & .35 & .30 & 1.00 & \\
\hline 8. Openness & .34 & .35 & .28 & .50 & .62 & .46 & .36 & 1.00 \\
\hline
\end{tabular}


mean structure. In doing so, we thought of our variables as reflecting different aspects of positive psychological functioning. In this conceptualization, it is the variance common to them all that captures the construct we believe to be important in the association between exercise and positive psychological functioning. That is, it is not, for example, extraversion per se that is associated with exercise. Rather, it is the surgency of positive mood and active engagement in life that often drive the overt sociability that our extraversion measure taps that is associated with exercise, and analogous statements can be made for all the eight variables that make up our latent variable.

A factor model of this kind allows for the calculation of model parameters in each group. We set one factor loading in the first group to 1.00 and the factor variances equal to identify the model. To establish that measurement of the involved constructs is consistent from group to group and thus that any observed difference in the latent factor means actually indicates a group difference, it is necessary that it be possible to constrain factor loadings, intercepts, and residual variances equal across groups without significant loss of model fit (Meredith, 1993). It was possible to do so in this case $\left(\Delta \chi^{2}=8.4\right.$ on $\left.23 d f, p=.99\right)$. The model parameter estimates are shown in Table 3 . The table shows that each measure of psychological functioning had substantial loadings on the general factor. The latent factor mean for the exercising group was set at 0 in establishing the model; that for the nonexercising group was freely estimated at -.31 . This difference was significant ( $p=.03$, two-tailed). The factor variance for each group was .51, indicating that the latent variable had about half as much variance as the eight

Table 3

Parameter Estimates from Factor Model with Two Groups Exercisers and Nonexercisers

\begin{tabular}{lcc} 
& \multicolumn{2}{c}{ Standardized } \\
\hline Variable & $\begin{array}{c}\text { Factor } \\
\text { loading }\end{array}$ & $\begin{array}{c}\text { Residual } \\
\text { variance }\end{array}$ \\
\hline Good mood & .56 & .69 \\
Optimism & .58 & .67 \\
Control & .60 & .64 \\
Agency & .75 & .44 \\
Extraversion & .84 & .29 \\
Agreeableness & .59 & .65 \\
Conscientiousness & .40 & .84 \\
Openness & .72 & .49 \\
Factor variance: & & .51 \\
Factor mean, MZ exercisers & .00 & \\
Factor mean, MZ nonexercisers & -.31 & \\
Significance of difference & .03 & \\
Standardized effect size & .43 & \\
\hline
\end{tabular}

Note: $\mathrm{MZ}=$ monozygotic. There were 63 pairs of $\mathrm{MZ}$ twins discordant for level of exercise. All parameters with the exception of the factor mean were constrained equal in the exercising and nonexercising groups. standardized variables underlying it. This factor variance meant that the mean difference had an effect size of $.43(.31 /$ square root of .51$)$.

We carried out several tests of the consistency of the latent factor mean difference in psychological functioning we observed between exercisers and nonexercisers. First, we wanted to establish that the difference we observed in the sample of discordant twins was not the result of some odd combination of circumstances in an unusual group of twins. The remainder of our sample consisted of both $\mathrm{MZ}$ and DZ twins who were not discordant for level of exercise. A median split on the exercise variable in this group (without regard to pair status) produced a latent factor mean difference with an effect size of .37 ( $p=.01$, two-tailed), indicating a very similar effect in exercisers and nonexercisers without co-twin control. Second, we wanted some evidence that it was the discordance for level of exercise rather than some difference related to the assignment of twin numbers or simply the separation of pairs that drove the latent mean difference in psychological functioning. The latent mean difference in MZ twins concordant for level of exercise had an effect size of -.09 , which was not significant ( $p=.49$, two-tailed). Third, we wanted to demonstrate that the effect held in the full sample of MZ twins, whether or not they met our definition of discordance for level of exercise. In the full sample of MZ twins, the correlation between the pair difference in level of exercise and the pair difference in psychological function latent variable factor scores was $.21(p<.001)$, indicating that the effect did hold throughout the sample of MZ twins.

Finally, though our data were contemporaneous, making inference of causal attribution impossible, we tabulated some data related to the direction of effect. More substantive differences in psychological functioning between twins discordant for exercise than differences in exercising between twins discordant for positive psychological functioning would suggest that exercise drives the improvement in psychological functioning rather than vice versa. To evaluate this, we used the mean differences in positive psychological functioning between exercisers and nonexercisers as boundaries to identify MZ twins discordant for positive psychological functioning. This yielded 58 pairs in which one member of the pair scored above the mean on positive psychological functioning for exercising twins in the group discordant for exercising, and the other member of the paired scored below the mean for nonexercising twins. This formed a group of twins discordant for level of psychological functioning; they had a mean difference with an effect size of .47. The lower functioning group reported exercising on average five times per month and the higher functioning group reported exercising on average seven times per month. Given the categories into which our data had been separated, this meant that distinction between the groups was only possible because we had 
averaged over the summer and winter reports. Thus, an $\mathrm{MZ}$ twin difference in psychological functioning comparable to that for exercise yielded little difference in level of exercise, hinting that the preponderant direction of effect is from exercise to positive psychological functioning. Corroborating this, only nine pairs, or about $15 \%$, were members of both the group discordant for exercise and the group discordant for level of psychological functioning.

The association between exercise and positive psychological functioning could also result from selection effects: there may be genetic influences common to both positive psychological functioning and involvement in exercise. To test this, we made use of the co-twin control method. In this analysis, the discordant twins served as quasi-experimental controls for each other in comparing mean levels of positive psychological functioning by level of exercise. Greater association between level of exercise and positive psychological functioning in DZs than in MZs, as measured by a significant interaction term involving zygosity and level of exercise, would provide evidence for genetic influences common to both positive psychological functioning and involvement in exercise. The interaction term, however, was not significant $(p=.51)$.

\section{Discussion}

This study made use of a sample of MZ twin pairs discordant for level of vigorous exercise to investigate the psychological benefits associated with regular exercise. Because prior investigations of such benefits have produced results that have varied from study to study with the specific measures of psychological functioning used, we made use of a latent variable reflecting the variance common to eight measures of psychological functioning. As has been the case in prior studies, exercise was not associated with better functioning on all of our individual measures. The latent variable, however, did show a significant mean difference between the two groups, with an effect size in excess of .4. This finding is important in developing an understanding of the ways that regular exercise contributes to maintenance of physical health. This may be especially true in aging populations, as exercise may reduce the rate of physical deterioration with age, and thus vulnerability to both mental and physical age-related diseases.

Evidence for the specific mechanisms linking brain and body is just now emerging. For example, it is becoming clear that the presence of depressive symptoms (or the absence of the kind of positive psychological functioning we measured in this study) contributes to the development of excessive inflammation or immune response, which in turn can contribute to the development of cardiac and autoimmune diseases (Miller \& Blackwell, 2007). Moreover, depressive symptoms precede the development of Alzheimer's disease, although it is not known whether such symptoms are an early manifestation of the disease, or play some causal role in the development of the disease. The relations between brain and body may be particularly important in aging groups, as all of the body's systems become less resilient with age. To the extent that exercise helps to maintain physical and/or psychological resilience, it may be particularly helpful in maintaining overall quality of life at older ages. These are questions for future research. Addressing these questions using longitudinal studies that allow the observation of how the importance of exercise in maintaining physical and psychological health and the links between them change with age will clearly be important.

Although our study did have an important finding that generates important questions for future research, it is also subject to some limitations. First, although national in scope, our sample is somewhat wealthier, better educated, Caucasian, and female than the United States as a whole. This makes generalization of our results to the full population of the United States difficult and makes inferences about the applicability of the psychological benefits of exercise that we observed to populations in other parts of the world tenuous. Second, the measures we used were all derived from self-report in a survey questionnaire format, a rather weak way of measuring exercise in particular. We have no way of knowing, for example, the actual intensity of exercise engaged in by participants who reported that they exercised vigorously, or whether these participants experienced measurable improvements in physical fitness. In addition, it is possible that, because they believed that exercise should make them feel better, our exercising participants reported that it did. Because we thought this might affect perceptions of life satisfaction most directly, we avoided such measures in our construct of positive psychological functioning.

Third, our data are contemporaneous rather than predictive, making it impossible to draw inferences about the causal nature of the associations we have observed. That is, it remains possible that those with higher positive psychological functioning were more likely to engage in vigorous exercise, while those with lower positive psychological functioning shied away from exercise. This would be an example of the wellknown epidemiological 'healthy worker effect' (Li \& Sung, 1999). We were, however, able to provide some information suggesting that the preponderant direction of effect flows from exercise to positive psychological functioning, and there was no explicit evidence for genetic influences common to positive psychological function and exercise. It would be valuable in future work to assess the degree to which psychological functioning changes in MZ twins who are initially concordant for little exercise when only one is randomly assigned to begin an exercise program, as well as to determine if there are psychological markers that predict which of such a pair of 
concordant $\mathrm{MZ}$ twins might undertake an exercise program. These questions, however, can only be addressed using longitudinal data. Finally, the size of our sample of $\mathrm{MZ}$ twins discordant for level of exercise was rather small at 63 pairs, limiting our power to detect effects.

In spite of these limitations, our study has some important strengths. The control for genetic and childhood environmental background provided by our sample of $M Z$ twins is very general - it encompasses all physical and behavioral variables influenced by those backgrounds, whether we can specify them or not. In addition, our use of a latent factor model made it possible to assess positive psychological functioning at a very general level without measurement error.

The variance common to our measures of psychological functioning reflects a positive, expansive sense of capacity to cope with life. Our evidence that exercise contributes to this sense is consistent with Salmon's (2001) theory of exercise training as stress adaptation. Drawing on Lees and Dygdon's (1988) argument that exercise can initially be unpleasant but can over time become pleasant due to counterconditioning, or the suppression of an unwanted response to a stimulus by the conditioning of a competing (e.g., endorphin) response, Salmon (2001) noted that the effects of counter-conditioning routinely extend beyond the originally aversive stimulus in nonhuman animal experiments so that counter-conditioning becomes a more general form of 'toughening up' (Gray, 1982). For example, after developing tolerance to exposure to cold water through counter-conditioning, animals also display tolerance to uncontrollable electric shock, and vice versa (Weiss et al., 1975).

In nonhuman animals, this kind of toughening up reflects not just reduced sensitivity or greater resilience to stress, but also reduced anxiety and depression as evidenced by the similarity of effects to those of anxiolytic and antidepressant drugs (Gray, 1982). Investigations of the specific neurochemical pathways involving serotonin, norepinephrine, and the endogenous opioid systems are in process (Salmon, 2001). We thus speculate that experience of the tougheningup process involved in engagement in vigorous exercise may generalize to other areas of life, providing the psychological resources needed to exert more effective control of emotional, relational, and intellectual as well as physical areas of life. In addition, we speculate that the toughening-up process involved in engagement in vigorous exercise may be one way to develop the psychological characteristic fundamentally underlying resilience (Ciccehetti \& Garmezy, 1993; Masten et al., 1999).

This theory about the psychological benefits of exercise and evidence supporting it may contribute to our understanding of the way in which exercise benefits physical health as well. McEwen (1993; McEwen $\&$ Wingfield, 2003) has suggested that organisms cope with stress through the adaptive process of allostasis, or the active maintenance of physiological stability through the production of hormones, neurotransmitters, and cytokines that regulate metabolic, cardiovascular, and immune responses. Regulation of this process of allostasis takes place in the brain (McEwen, 2004). When allostasis is activated too often or inefficiently managed, individuals are more vulnerable to stress-related physical diseases. The counter-conditioning involved in generating reduced sensitivity or greater resilience to stress as a result of vigorous exercise may be the very mechanism that enables the individual to maintain better allostatic balance and resist physical illness, thus linking brain, body, and behavior.

The particular form of our study highlights an important aspect of these psychological and physical counter-conditioning processes, to the extent they exist. Because we made use of MZ twins, we know that the differences in psychological functioning we observed cannot be attributed to genomic differences. At the same time, the specific mechanisms hypothesized to be involved in the counter-conditioning processes are highly biological and thus must also involve genetic action. It is very likely that the apparent contradiction between an absence of genomic differences and differences in manifestation of phenotypes that depend on genetic action will be resolved through understanding how genetic expression varies across different social and personal contexts of physical exercise. Our results thus serve as an inspiration for future sociogenomic research.

\section{Acknowledgments}

This research was supported by the John D. and Catherine T. MacArthur Foundation Research Network on Successful Midlife Development, and by National Institute on Aging Grant \#AG20166. Address correspondence to Wendy Johnson at john4350@tc.umn.edu or Robert Krueger at krueg038@tc.umn.edu, Department of Psychology, 75 East River Road, Minneapolis, MN 55455.

The authors acknowledge funding support from the National Institute on Aging. The views expressed are those of the authors of this paper, and not the National Institute on Aging, the National Institutes of Health or the Department of Health and Human Services.

\section{References}

Annesi, J. J., \& Unruh, J. L. (2004). Effects of a treatment protocol on the drop-out rates of exercise participants in 17 YMCA facilities in 6 cities. Psychological Reports, 95, 250-256.

Bollen, K. A. (1989). Structural equations with latent variables. New York: John Wiley \& Sons.

Bouchard, T. J., Jr., \& Loehlin, J. (2001). Genes, evolution, and personality. Behavior Genetics, 31, 243-273. 
Brawley, L. R., \& Rodgers, W. M. (1994). Social-psychological aspects of fitness promotion. In P. Seraganian (Ed.), Exercise psychology: The influence of physical exercise on psychological processes (pp. 254-298). New York: Wiley.

Ciccehetti, D., \& Garmezy, N. (1993). Prospects and promises in the study of resilience. Development and Psychopathology, 5, 497-502.

Colcombe, S. J., Erickson, K. I., Scalf, P. E., Kim, J. S., Prakash, R., McAuley, E., Marquez, D. X., Hu, L., \& Kramer, A. F. (2006). Aerobic exercise training increases brain volume in aging humans. Journals of Gerontology, Series A: Biological Science and Medical Science, 61, 1166-1170.

Dishman, R. K., Berthoud, H. R., Booth, F. W., Cotman, C. W., Edgerton, G. R., Fleshner, M. ,R., Gandevia, S. C., Gomez-Pinilla, F., Greenwoord, B. N., Hillman, C. H., Kramer, A. F., Levin, B. E., Moran, T. H., RussoNeustadt, A. A., Salamone, J. D., Van Hoomissen, J. D., Wade, C. E., York, D. A., \& Zigmond, M. J. (2006). Neurobiology of exercise. Scandinavian Journal of Medical Science and Sports, 16, 470.

Fletcher, G. F., Blair, S. N., Blumenthal, J., Casperson, C., Chaitman, B., Epstein, L., Fall, H., Froelicher, V. F., \& Pina, I. L. (1992). Statement on exercise: Benefits and recommendations for physical activity programs for all Americans. Circulation, 86, 340-344.

Girodo, M., \& Pellegrini, W. (1976). Exercise-produced arousal, film-induced arousal, and attribution of internal states. Perceptual and Motor Skills, 42, 931-935.

Goldberg, L. R. (1992). The development of markers for the Big-Five factor structure. Psychological Assessment, $4,26-42$.

Gray, J. A. (1982). The Neuropsychology of anxiety: Enquiry into the function of the septohippocampal system. Oxford, UK: Clarendon Press.

Hamdorf, P., Starr, G., \& Williams, M. (2002). A survey of physical-activity levels and functional capacity in older adults in South Australia. Journal of Aging and Physical Activity, 10, 281-289.

Haskell, W. L. (1984). Overview: Health benefits of exercise. In J. D. Matarazzo, S. M. Weiss, J. A. Herd, N. E. Miller, \& S. W. Weiss (Eds.), Behavioral health: A handbook of health enhancement and disease prevention (pp. 409-423). New York: J. Wiley.

John, O. P. (1990). The 'Big Five' factor taxonomy: Dimensions of personality in the natural language and in questionnaires. In L. A. Pervin (Ed.), Handbook of personality: Theory and research (pp. 66-100). New York: Guilford Press.

Johnson, W., \& Krueger, R. F. (2004). Genetic and environmental structure of adjectives describing the Big Five model of personality: A nationwide twin study. Journal of Research in Personality, 38, 448-472.

Kartodihardjo, W., \& Klissouras, V. (1976). Growth and physical training with reference to heredity. Journal of Applied Physiology, 40, 211-215.
Kessler, R. C., Gilman, S. E., Thornton, L. M., \& Kendler, K. S. (2004). Health, wellbeing, and social responsibility in the MIDUS twin and sibling subsamples. In O. G. Brim, C. D. Ryff, \& R. C. Kessler (Eds.), How healthy are we? A national study of wellbeing at midlife (pp. 124-152). Chicago: University of Chicago Press.

Lachman, M. E., \& Weaver, S. L. (1997). The Midlife Development Inventory (MIDI) personality scales: Scale construction and scoring. Waltham, MA: Brandeis University.

Lees, L. A., \& Dygdon, J. A. (1988). The initiation and maintenance of exercise behavior: A learning theory conceptualization. Clinical Psychology Review, 8, 345-353.

Li, C. Y., \& Sung, F. C. (1999). A review of the healthy worker effect in occupational epidemiology. Occupational Medicine (Lond), 49, 225-229.

Masten, A. S., Hubbard, J. J., Gest, S. D., Tellegen, A., Garmezy, N., \& Ramirez, M. (1999). Competence in the context of adversity: Pathways to resilience and maladaptation from childhood to late adolescence. Development and Psychopathology, 11, 143-169.

McEwen, B. S. (1993). Stress and the individual: Mechanisms leading to disease. Archives of Internal Medicine, 153, 2093-2101.

McEwen, B. S. (2004). Allostasis and allostatic overload and relevance to the pathophysiology of psychiatric disorders. Annals of the New York Academy of Sciences, 1032, 1-7.

McEwen, B. S., \& Wingfield, J. C. (2003). The concept of allostasis in biology and biomedicine. Hormones and Behavior, 43, 2-15.

McIntyre, C. W., Watson, D., \& Cunningham, A. C. (1990). The effects of social interaction, exercise, and test stress on positive and negative affect. Bulletin of the Psychonomic Society, 28, 141-143.

Meredith, W. (1993). Measurement invariance, factor analysis, and factorial invariance. Psychometrika, 58, 525-543.

Miller, G. E., \& Blackwell, E. (2007). Turning up the heat: Inflammation as a meachanism linking chronic stress, depression, and heart disease. Current Directions in Psychological Science, 15, 269-272.

Perusse, L., Rankinen, T., Rauramaa, R., Rivera, M. A., Wolfarth, B., \& Bouchard, C. (2003). The human gene map for performance and health-related fitness phenotypes: The 2002 Update. Medicine and Science in Sports and Exercise, 35, 1248-1264.

Petruzello, S. J., Jones, A. C., \& Tate, A. K. (1997). Affective responses to acute exercise: A test of opponent process theory. Journal of Sports Medicine and Physical Fitness, 37, 205-212.

Raglin, J. S. (2001). Factors in exercise adherence: Influence of spouse participation. Quest, 53, 356-361.

Rouselle, J. G., Blascovich, J., \& Kelsey, R. M. (1995). Cardiorespiratory response under combined 
psychological and exercise stress. International Journal of Pschophysiology, 20, 49-58.

Salmon, P. (2001). Efects of physical exercise on anxiety, depression, and sensitivity to stress: A unifying theory. Clinical Psychology Review, 21, 33-61.

Simonen, R. L., Perusse, L., Rankinen, T., Rice, T., Rao, D. C., \& Bouchard, C. (2002). Familial aggregation of physical activity levels in the Quebec Family Study. Medicine and Science in Sports and Exercise, 34, 1137-1142.

Steptoe, A., Kearsley, N., \& Walters, N. (1993). Acute mood response to maximal and submaximal exercise in active and inactive men. Psychology and Health, 8, 89-99.
Thompson, P. D., Tsongalis, G. J., Ordovas, J. M., Seip, R. L., Bilbie, C., Miles, M., Zoeller, R., Visich, P., Gordon, P., Angelopoulos, T. J., Pescatello, L., \& Moyna, N. (2006). Angiotensin-converting enzyme genotype and adherence to aerobic exercise training. Preventive Cardiology, 9, 21-24.

Trapnell, P. D., \& Wiggins, J. S. (1990). Extension of the interpersonal adjective scales to include the Big Five dimensions of personality. Journal of Personality and Social Psychology, 59, 781-790.

Weiss, J. M., Glazer, H. I., \& Miller, N. E. (1975). Effects of chronic exposure to stressors on subsequent avoidance-escape behavior and on brain norepinephrine. Psychosomatic Medicine, 37, 522-534. 